\title{
Reconstrucción microquirúrgica del velo del paladar y pared lateral de orofaringe. Una nueva propuesta de clasificación. Resultados funcionales*
}

\author{
Dr. ALVARO COMPAN J., \\ Istituto Nazionale per la cura e lo Studio dei Tumori. Milano, Italia. \\ 2 Hospital Clínico Regional de Concepción, Universidad de Concepción, Concepción, Chile.
}

\section{TRABAJO DE INGRESO}

\begin{abstract}
Microsurgical velopharyngeal reconstruction. Evaluation of 37 patients

Background: Velopharyngeal reconstruction after surgical excision of tumors is complex and must preserve swallowing and phonation Aim: To assess quality of life of patients subjected to velopharyngeal reconstruction after excision of a pharyngeal carcinoma. To propose a new classification velopharyngeal junction defects, based on tridimensional criteria, to allow a reconstruction using radial forearm flaps. Material and Methods: Prospective evaluation of 37 patients aged 25 to 78 years, with a carcinoma that involved the lateral wall of the oropharynx or soft palate, subjected to surgical excision with negative surgical margins. Four types of velopharyngeal effects were considered for reconstruction. In 25 of the patients quality of life and quality of swallowing and phonation was evaluated. Results: Eighty one percent of patients considered their quality of life as good or optimal. No patients had swallowing disturbances and 71,21 and $8 \%$ had an excellent, good or acceptable phonetic results, respectively. Conclusions: The proposed classification allows a reconstruction without tension and with a good functional performance.
\end{abstract}

Key words: Soft palate reconstruction, oropharyngeal cancer, quality of life.

\section{Resumen}

El propósito de esta serie clínica es la de proponer una clasificación de los defectos de la unión velofaríngea y/o pared lateral de la orofaringe basados en criterios tridimensionales que permita una reconstrucción con colgajos libres radiales de antebrazo. Se evalúa además la calidad de vida, lenguaje y deglución. Métodos: Estudio de tipo prospectivo, longitudinal que seleccionó 37 pacientes. La clasificación reconstructiva propuesta se basó en un criterio de tipo geométrico-espacial que distinguió 4 tipos de defectos. Los datos obtenidos se llevaron a tablas y se realizó análisis mediante frecuencias y promedios.

\footnotetext{
*Recibido el 24 de Marzo de 2008 y aceptado para publicación el 27 de Enero de 2009.
}

Correspondencia: Dr. Alvaro Compan J.

Chacabuco esq. Janequeo, $4^{\circ}$ Piso, Concepción, Chile.

E-mail: alcompan@gmail.com 
Resultados: Al aplicar los criterios de inclusión sólo 37 (47,43\%) de los 78 pacientes cumplieron con ellos. La calidad de vida aplicando la encuesta de Gliklich, se efectuó a 25 pacientes $(67,56 \%)$ de un total de 28 vivos. El estudio reveló que el $81 \%(\mathrm{n}=20)$ refería tener una calidad de vida buena u óptima. El 100\% $(\mathrm{n}=25)$ de los pacientes no mostró dificultad en la deglución, sólo se observó prolongación en los tiempos de las fases oral y faríngea en cinco pacientes. El control fonoaudiológico mostró que un $71 \%$ de los pacientes presentó un resultado fonético excelente, $21 \%$ fue bueno y un $8 \%$ aceptable. Conclusiones: La clasificación propuesta permite establecer una correlación entre el defecto espacial y el modelo reconstructivo permitiendo de esta forma una reconstrucción sin tensiones y con un buen resultado deglutorio, fonético y de calidad de vida.

Palabras clave: Unión velofaríngea, cirugía, reconstrucción.

\section{Introducción}

Las reconstrucciones necesarias, después de realizadas resecciones oncológicas de la unión velo faríngea y/o pared lateral de la orofaringe, presentan grandes complejidades y desafíos técnicos, ya que la región a reconstruir posee una superficie tridimensional de características extremadamente particulares que involucra a importantes funciones tales como la deglución y la fonación; si bien existen varias modalidades reconstructivas son sin duda los colgajos libres los que producen los mejores resultados funcionales que impactan directamente la calidad de vida de los pacientes.

Dentro de la infinidad de colgajos libres ${ }^{1,2}$, aquellos que son útiles en esta región deben tener cualidades especiales tales como un pedículo de longitud adecuada, un grosor y una capacidad de doblarse sobre sus ejes espaciales sin sufrir isquemia o congestión venosa ${ }^{3,4}$. Es por esto que el colgajo elegido que posee todos estos atributos es el colgajo libre radial de antebrazo ${ }^{5,6}$.
En la literatura existen intentos de clasificación de estos defectos, debido a la ausencia de una clasificación histórica. Algunas de éstas sugieren que las soluciones de continuidad post quirúrgicas deben tratarse en base al tamaño de la resección y para esto se han propuesto modelos reconstructivos basados en criterios bidimensionales (Figura 1) que no tienen en cuenta la particular conformación anatómica de la región y que habitualmente modifican la anatomía de los tejidos remanentes creando tensiones inadecuadas entre éstos y los colgajos libres generando así un mayor número de dehiscencias y complicaciones.

En este sentido el Dr. Kimata ${ }^{6}$ ha sido pionero en sistematizar los defectos en tres tipos, clasificándolos en Tipo I aquellos que comprometen la pared lateral de la orofaringe, Tipo II con extensión a la pared orofaringea superior y Tipo III defectos con extensión contralateral.

En la nueva clasificación propuesta no se toma en cuenta el compromiso contralateral del velo ni la línea media; sin embargo, resulta más importante

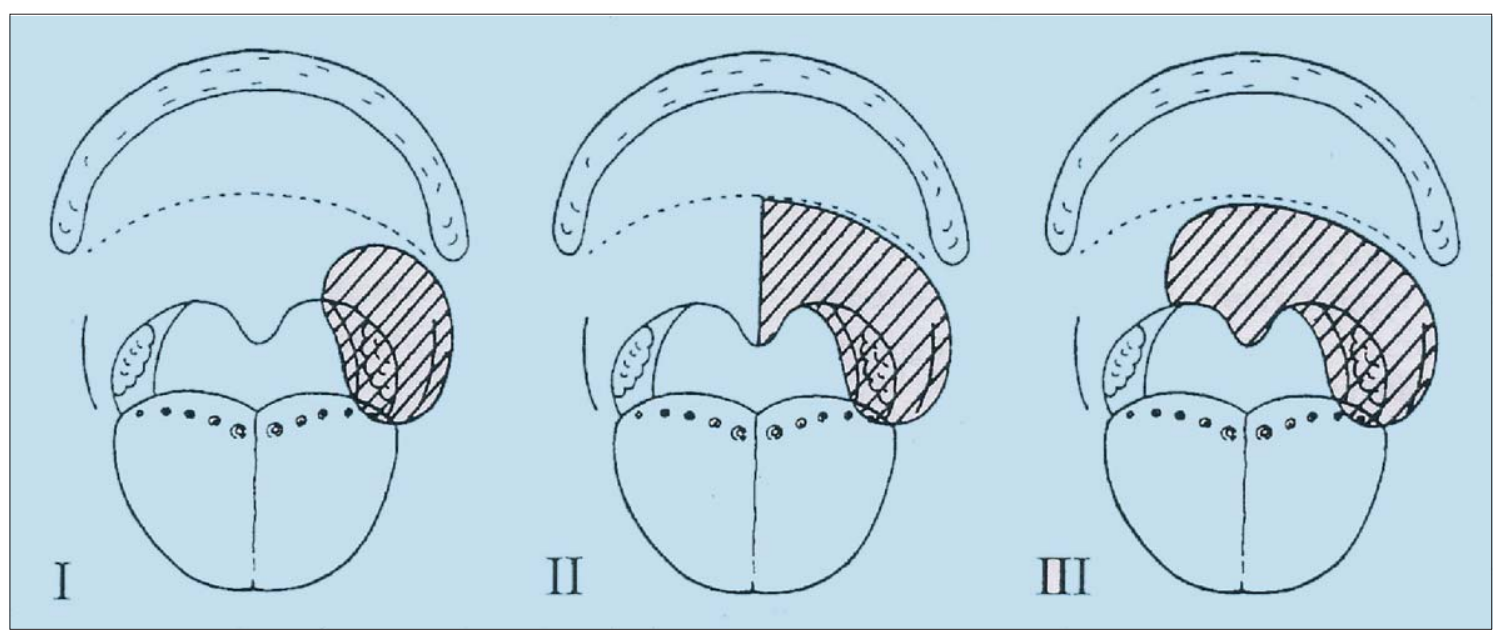

Figura 1. Clasificación de los defectos velotonsilares según Kimata. 
que tomar en consideración estos elementos, pensar en el defecto creado tridimensionalmente, es decir, en forma espacial, ya que lo realmente útil son las líneas de tensión que se generan entre el colgajo y el sitio a reconstruir ${ }^{5,7}$.

La conformación tridimensional tiene un asidero espacial al pensar que cada pared del sitio a reconstruir corresponde a la cara de un cuerpo geométrico. La clasificación propuesta se basa en este punto; por ejemplo la división de los defectos Tipo 4 en a y b no es aleatoria ya que desde el punto de vista reconstructivo es importante definir si los defectos a reparar son anterolaterales o posterolaterales, punto que determinará el tipo de diseño del colgajo y su posicionamiento sobre la solución de continuidad creada, e incidirá directamente en las líneas de tensión que se generen durante el proceso de cicatrización, especialmente al realizar modificaciones al colgajo libre radial de antebrazo (colgajo tipo "P"). No resulta lógico entonces reconstruir un sitio tridimensional con un colgajo libre que considere sólo criterios en dos dimensiones.

El presente trabajo, tiene por finalidad aportar un nuevo criterio que es de la tridimensionalidad de la región, que hasta el momento no se había considerado en ninguna de las clasificaciones y que, resulta vital al momento de la reconstrucción ya que permite colgajos viables sin tensión.

La forma de estudiar el problema reconstructivo, fue mediante el diseño de una serie prospectiva de casos que tuvo como objetivo evaluar la clasificación quirúrgica propuesta y asociarla con un procedimiento reconstructivo, midiendo sus resultados en el tiempo y correlacionándola con calidad de vida, fonación y deglución.

\section{Objetivos}

\section{Dos son los objetivos de este trabajo}

El primer objetivo, es proponer una nueva clasificación de los defectos de la unión velofaringea y/o pared lateral de la orofaringe, basados en criterios tridimensionales que eviten tensiones inadecuadas entre el colgajo libre y los tejidos remanentes de ésta.

El segundo objetivo, es evaluar calidad de vida, preservación del lenguaje y de la función deglutoria posterior a la reconstrucción con colgajo libre radial de antebrazo siguiendo los criterios tridimensionales de acuerdo a la clasificación propuesta.

\section{Material y Método}

Estudio de tipo prospectivo, longitudinal que seleccionó 37 pacientes portadores de carcinoma espinocelular que comprometían la pared lateral de la orofaringe y/o el velo del paladar y a los cuales se les realizó una resección oncológica con bordes quirúrgicos negativos (Ro) en el Servicio de Cirugía Cráneo maxilo facial del Istituto Nazionale per la cura e lo Studio dei Tumori. Milano, Italia y el Hospital Clínico Regional de Concepción, Chile. Se realizaron 34 cirugías en Italia y 3 en Chile, efectuadas por dos equipos quirúrgicos en las cuales el autor participó como cirujano y ayudante. La técnica quirúrgica como asimismo todos los procedimientos terapéuticos y diagnósticos fueron informados oportuna y claramente a cada paciente y sus familiares, cumpliendo con la legislación en ambos países donde se realizaron los estudios. Se firmaron los consentimientos requeridos por las leyes correspondientes de cada país.

La reconstrucción se realizó durante el mismo tiempo quirúrgico utilizando colgajos libres radiales de antebrazo.

La selección de los pacientes se basó en los siguientes criterios:

\section{Criterios de Inclusión}

- Carcinomas espinocelulares resecables de la pared lateral de la orofaringe y/o velo del paladar.

- Pacientes sin ningún tipo de tratamiento previo (Radioterapia, Quimioterapia).

- Defectos tipo 2, 3 y 4 de la clasificación propuesta.

- Monofocalidad.

El diagnóstico histopatológico inicial se realizó mediante biopsia incisional de la lesión bajo sedación anestésica en pabellón esperando confirmación histológica diferida.

El estudio diagnóstico inicial consideró para todos los pacientes TAC de cavidad oral contrastados con reconstrucción $3 \mathrm{D}$, cuello, tórax y nasofibroscopia.

La resecabilidad tumoral se definió en términos de la escisión quirúrgica completa excluyendo aquellos tumores que presentaron invasión laríngea, invasión de la musculatura de base lingual, invasión ósea mandibular, infiltración carotídea o compromiso de base de cráneo.

El status linfonodal del cuello no fue considerado para el desarrollo de la técnica.

La clasificación reconstructiva propuesta se basó en un criterio de tipo geométrico-espacial que distinguió 4 tipos de defectos. Esto es, el defecto generado determina el tipo de reconstrucción con un colgajo libre radial de antebrazo (Figura $2 \mathrm{a}$ y $2 \mathrm{~b}$ ).

Tipo 1: Defectos del velo limitados que no requieren una reconstrucción microquirúrgica.

Tipo 2: Defectos monoplanares velo tonsilares que se desarrollan sobre el plano frontal. 
Clasificación de los defectos velo-faríngeos y/o Pared Lateral de Orofaringe

Tipo 1 Defectos Velares limitados al velo del Paladar

Tipo 2 Defectos monoplanares velo-tonsilares

Tipo 3 Defectos monoplanares Laterales

Tipo 4a Defectos Multiplanares Anterolaterales

Tipo 4b Defectos Multiplanares Posterolaterales

Figura 2a. Clasificación de los defectos.

TIPO 1.

Defectos limitados al velo del paladar

TIPO 2.

Defectos moplanares velotonsilares

TIPO 3.

Defectos monoplanares

laterales

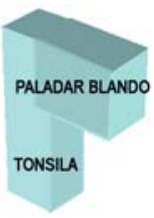

TIPO 4a.

Defectos multiplanares velofaringeos anteriores

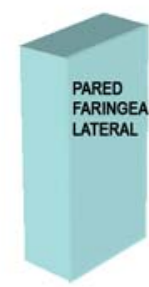

TIPO 4b.

Defectos multiplanares velofaringeos posteriores
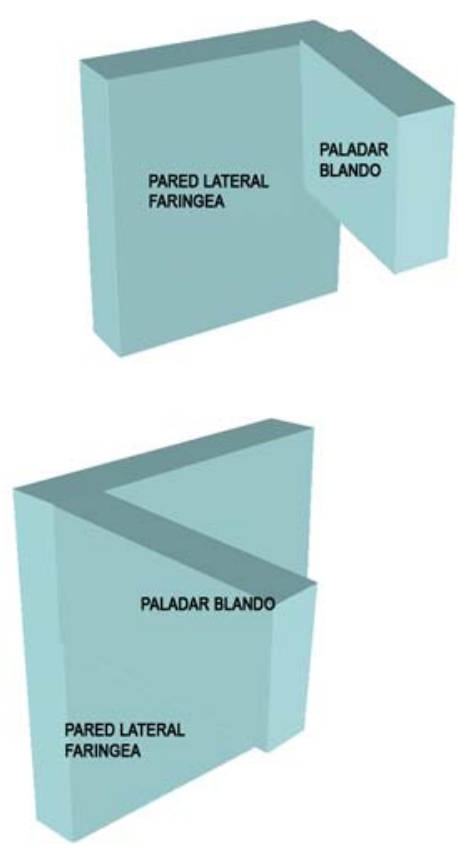

Figura 2b. Clasificación espacial propuesta de acuerdo al defecto creado post resección. 
Tipo 3: Defectos monoplanares de la pared lateral de la orofaringe dispuestos en plano sagital.

Tipo 4: Defectos multiplanares que comprenden el paladar blando y la pared lateral faríngea anterior (tipo 4a) o posterior (tipo 4b), es decir, defectos que comportan la combinación de dos planos espaciales.

El método reconstructivo se pudo aplicar a todos los defectos utilizando la nueva clasificación, se exceptuaron los defectos tipo 1 por no requerir una reconstrucción con colgajos libres.

Los defectos Tipo 2 se repararon mediante un colgajo radial rectangular clásico plegado sobre sí mismo con el eje mayor sobre el paladar blando y el istmo de las fauces.

En los defectos Tipo 3 se utilizó un colgajo radial de antebrazo clásico de tipo rectangular posicionado sobre la pared lateral de la orofaringe.

En los defectos Tipo 4 se realizó una modifica- ción del colgajo radial de antebrazo denominado colgajo a "P" (Figuras 3a, 3b y 3c) en donde el brazo corto de la $\mathrm{P}$ se dobló sobre sí mismo para reconstruir el paladar blando y el brazo largo se dejó plano para reconstruir la pared lateral faríngea. $\mathrm{El}$ diseño de la $\mathrm{P}$ de forma especular permitió reparar todos los defectos tipo $4 \mathrm{a}$ y $4 \mathrm{~b}$, fueran derechos o izquierdos.

De manera de objetivar los resultados funcionales y su impacto en la calidad de vida de los pacientes se evaluaron tres aspectos: calidad de vida, fonación y deglución.

\section{a) Calidad de Vida (Gliklich)}

En 25 pacientes de la serie se evaluó la percepción de la calidad de vida (QOL) y su relación con la reconstrucción practicada según la nueva clasificación propuesta para lo cual se aplicaron los cuatro criterios básicos determinados por el Dr. Gliklich

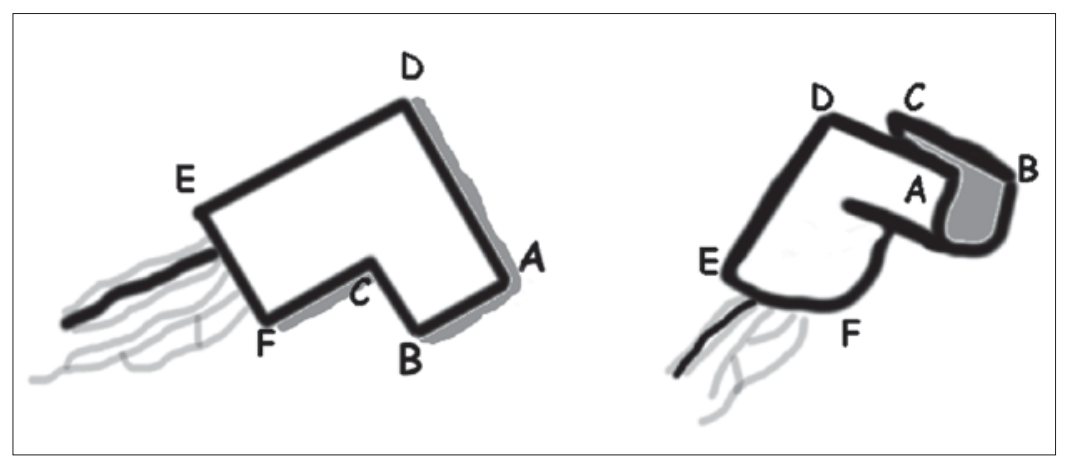

Figura 3a. Diseño de colgajo libre radial de antebrazo modificado a "P" para reconstrucciones Tipo 4.

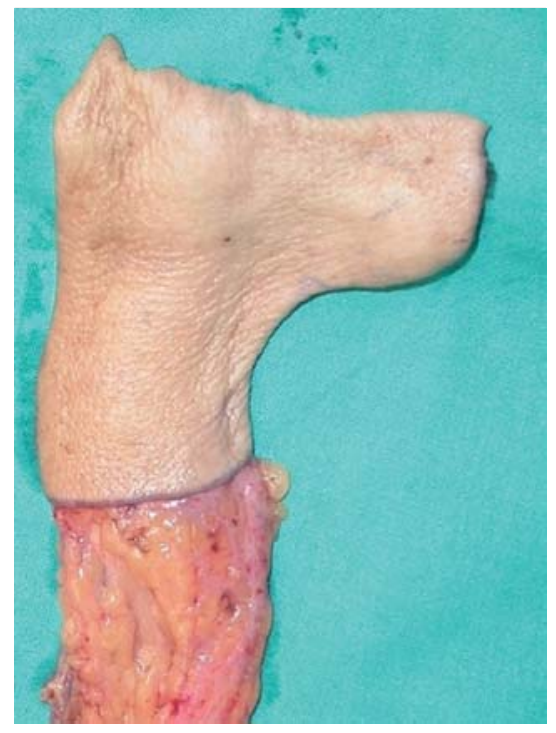

Figura 3b. Colgajo radial tipo "P" para reconstrucciones Tipo 4.

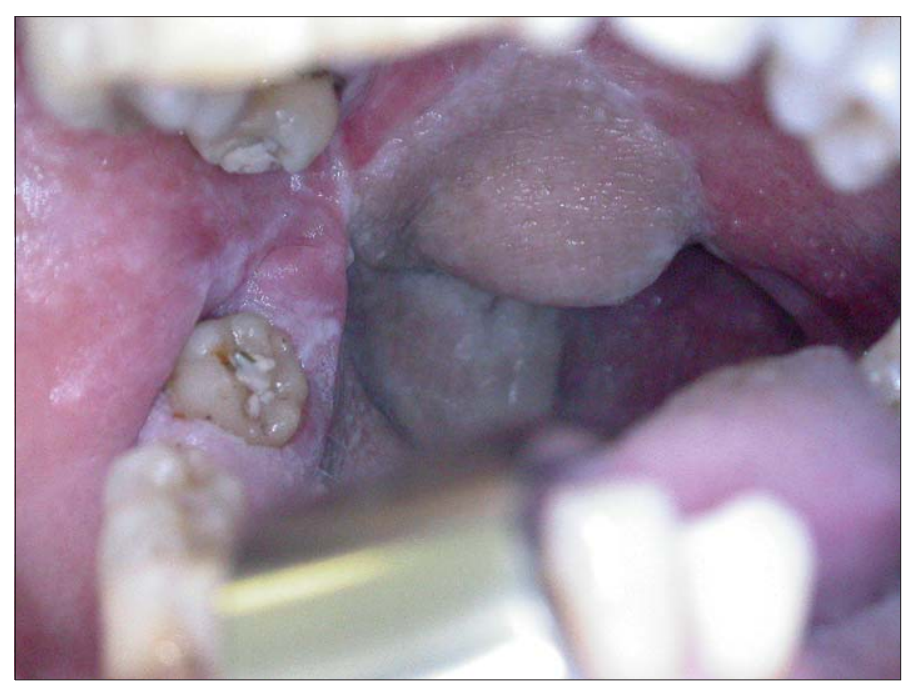

Figura 3c. Defecto Tipo 4 reconstruido con colgajo radial tipo "P". 
$\mathrm{RE}^{8}$, derivados del análisis y entrecruzamiento de datos de 4 sistemas de evaluación de calidad de vida (UW-QOL v 4.0, SF-36 Health Survey, the H\&NS y el PSS-HN). Se seleccionaron 4 dominios: dolor, alimentación, lenguaje y aspecto, que fueron aquellos seleccionados por Gliklich y que corresponden a los parámetros de evaluación de calidad de vida en pacientes con patología de cabeza y cuello oncológica. Estos criterios fueron traducidos del inglés al italiano y al español.

Los criterios de Gliklich no corresponden a una clasificación propiamente tal y por tanto, no requirieron autorización para su utilización.

Para cada aspecto a evaluar se estableció una escala que varió de 0 (muy pobre) a 100 (excelente). Se obtuvieron resultados para cada ítem los cuales fueron llevados a una tabla de datos y analizados. Los resultados se expresaron en porcentajes.

La encuesta fue aplicada por el mismo encuestador (autor) el cual explicó en forma detallada el tenor de ésta al paciente y al mismo tiempo fueron consultados los comités de ética de ambas instituciones.

\section{b) Deglución}

La evaluación de la deglución se realizó mediante cinefluoroscopia ${ }^{7,-11}$. Se analizaron la fase oral y la fase faríngea, no interesaron para propósitos de este estudio la fase de preparación del bolo alimenticio y la fase esofágica. Cada paciente fue evaluado post operatoriamente un mes después de haber sido dado de alta.

Todos los pacientes fueron situados lateralmente para evaluar adecuadamente este plano. Cada paciente debió deglutir $10 \mathrm{ml}$ de bario líquido; $10 \mathrm{ml}$ de bario mezclado con puré de papas, de manera de generar un alimento semisólido y una galleta mezclada con bario que debió ser masticada y degluti$\mathrm{da}^{6}$, este procedimiento se realizó dos veces por cada paciente para determinar un promedio.

Se registraron en vídeo todas las fases a fin de establecer la duración de cada una de ellas y correlacionarlas con la situación normal, usando como medida de tiempo segundos. La fase oral se definió como el período desde el ingreso a la boca hasta antes del pasaje a la orofaringe. La fase faríngea fue definida desde la entrada a la orofaringe hasta el pasaje a nivel de la unión cricofaríngea.

Para el análisis de los datos se midieron los tiempos de tránsito para cada tipo de ingesta tabulándose posteriormente para su análisis.

\section{c) Fonación}

Un fonoaudiólogo en Italia y 1 en Chile evaluaron a los pacientes a los que se aplicó la encuesta de calidad de vida.
Los elementos fonéticos utilizados en Italia fueron "Aiuoli y Patacca", sin embargo, en español estos fonemas no existen. El grupo propuso la homologación a dos palabras, como válidos para esta serie en español similar al italiano que fueron "Ayuda y Petaca" debido a que fonéticamente tienen similitud en ambas lenguas y permiten comparar los resultados.

La evaluación de la función fonatoria se centró en la competencia del esfínter velofaríngeo post reconstrucción ${ }^{12}$. Se evaluó el escape nasal, hipernasalidad, articulación de los fonemas e inteligibilidad del discurso. Todos los parámetros fueron medidos por un fonoaudiólogo en Italia y otro en Chile, mediante el uso de frases predeterminadas utilizando como principal instrumento de medición el oído del examinador. Se creó una escala de resultados fonéticos:

- Excelente: Ninguna alteración del habla.

- Bueno: Hipernasalidad o alteración en la articulación de un fonema.

- Aceptable: Discurso inteligible, dificultad articulatoria en 2 fonemas y/o escape nasal.

- Malo: Discurso ininteligible.

Los datos se llevaron a una tabla para ser tabulados en porcentajes.

\section{Resultados}

Un total de 78 pacientes presentaron tumores de la unión velofaríngea y/o pared lateral de la orofaringe entre Enero 2003 y Febrero 2006. Al aplicar los criterios de inclusión 37 pacientes $(47,43 \%)$ los cumplieron.

La distribución por sexos fue $40 \%$ para el sexo femenino y de $60 \%$ para el sexo masculino. La distribución por edades varió entre los 25 y los 78 años, encontrándose la frecuencia mayor en el rango 4060 años correspondiendo al 56,75\% (Tabla 1).

Según la clasificación propuesta 23 casos pertenecieron al Tipo 3 y 10 al Tipo 4, de los cuales 7

Tabla 1. Distribución por edades

\begin{tabular}{lc}
\hline Edad & n \\
\hline$<30$ años & 1 \\
$31-40$ años & 3 \\
$41-50$ años & 10 \\
$51-60$ años & 11 \\
$61-70$ años & 8 \\
$>70$ años & 4 \\
Total & 37 \\
\hline
\end{tabular}


Tabla 2. Número de casos por tipo de defecto

\begin{tabular}{llr}
\hline Tipo defecto & n \\
\hline Tipo 1 & Defectos velares & 0 \\
Tipo 2 & Defectos monoplanares tonsilares & 4 \\
Tipo 3 & Defectos monoplanares laterales & 23 \\
Tipo 4a & Defectos multiplanares anterolaterales & 7 \\
Tipo 4b & Defectos multiplanares postero-laterales & 3 \\
\hline
\end{tabular}

fueron Tipo 4a y 3 Tipo 4b. Sólo 4 pacientes presentaron defectos Tipo 2 (Tabla 2).

\section{Evolución de los colgajos}

En relación a los defectos tipo 2 de la clasificación, se reconstruyeron 4 defectos que comprometieron sólo el velo del paladar; no se consideró el compromiso de línea media como tampoco el contralateral, debido a que la amplitud del colgajo radial de antebrazo permitió una adecuada reconstrucción sin generar tensión excesiva en el plano frontal, sólo dos colgajos presentaron congestión venosa que cedió a las 72 horas (Tabla 3).

Los defectos Tipo 3, es decir, aquellos monoplanares laterales que se disponían en el plano sagital y que comprometieron sólo la pared lateral, presentaron dehiscencia parcial en 4 y congestión en 5 . Isquemia y necrosis no se observó en ningún colgajo.

Los defectos anterolaterales Tipo 4a presenta- ron una dehiscencia en uno y en otro se observó congestión venosa.

Los defectos posterolaterales Tipo 4b, presentó en uno necrosis de un $20 \%$ de su superficie en tanto que los otros dos no tuvieron complicaciones.

Existió superposición de complicaciones en los defectos Tipo 3 y 4 a.

El análisis de la deglución, fonación y calidad de vida fue posible efectuarlo en 25 pacientes del total de la muestra $(n=37)$ lo que representó un $67,56 \%$, esto debido a que se constataron 9 defunciones $(5$ fallecidos por recidiva del primario y 4 por causa médica) y 3 pacientes no fue posible localizarlos por cambio de región.

\section{a) Evaluación de la Calidad de Vida}

La evaluación objetiva de calidad de vida aplicando la encuesta de Gliklich, se efectuó a 25 pacientes $(67,56 \%)$ de un total de 28 vivos un año después de realizada la cirugía.

La distribución por tipo de defecto creado en esta muestra fue de 3 pacientes para el Tipo 2, 17 pacientes Tipo 3, 3 pacientes Tipo 4a y 3 pacientes Tipo 4b.

El estudio reveló que el $81 \%(n=20)$ refería tener una calidad de vida buena u óptima. Al analizar los datos se obtuvo que $70 \%(\mathrm{n}=18)$ redujo la sintomatología dolorosa a niveles calificados como buenos u óptimos y $60 \%(n=15)$ mejoró su calidad de alimentación (Tabla 4). El lenguaje fue calificado como inteligible en 23 pacientes y el aspecto fue insuficiente en dos pacientes.

Tabla 3. Complicaciones de los colgajos libres radial de antebrazo

\begin{tabular}{lccccccc}
\hline & Dehiscencia & $\begin{array}{c}\text { Complicaciones } \\
\text { Necrosis } \\
\mathbf{2 0 \%}\end{array}$ & $\begin{array}{c}\text { Necrosis } \\
\mathbf{2 0 \% - 5 0}\end{array}$ & $\begin{array}{c}\text { Necrosis } \\
\mathbf{>} \mathbf{5 0 \%}\end{array}$ & $\begin{array}{c}\text { Congestión } \\
\text { venosa }\end{array}$ & $\begin{array}{c}\text { Isquemia } \\
\text { Sin } \\
\text { complicaciones }\end{array}$ \\
\hline Defectos Tipo 2 & 0 & 0 & 0 & 0 & 2 & 0 & 2 \\
Defectos Tipo 3 & 4 & 0 & 0 & 0 & 5 & 0 & 19 \\
Defectos Tipo 4a & 1 & & & & 1 & 5 \\
Defectos Tipo 4b & & 1 & & & & 2 \\
\hline
\end{tabular}

Tabla 4. Resultados de calidad de vida según encuesta de Gliklich

\begin{tabular}{lccccc}
\hline & $\begin{array}{c}<\mathbf{2 0 \%} \\
\text { Insuficiente }\end{array}$ & $\begin{array}{c}\mathbf{2 0 - 3 9 \%} \\
\text { Suficiente }\end{array}$ & $\begin{array}{c}\mathbf{4 0 - 5 9 \%} \\
\text { Discreto }\end{array}$ & $\begin{array}{c}\mathbf{6 0 - 7 9 \%} \\
\text { Bueno }\end{array}$ & $\begin{array}{c}>\mathbf{8 0 \%} \\
\text { Óptimo }\end{array}$ \\
\hline Dolor & 0 & 5 & 2 & 7 & 11 \\
Alimentación & 0 & 5 & 5 & 7 & 8 \\
Lenguaje & 0 & 2 & 0 & 13 & 10 \\
\hline
\end{tabular}




\section{b) Evaluación de la Deglución}

El estudio de la deglución se efectuó en 25 pacientes a los cuales se les aplicó la encuesta de calidad de vida de Gliklich. Se administró sulfato de bario observando la formación del bolo alimenticio que varió en consistencia de sólido a líquido.

El 100\% $(n=25)$ de los pacientes no mostró dificultad en la deglución, sólo se observó prolongación en los tiempos de las fases oral y faríngea (Tabla 5). La duración de esta función fue normal en el $80 \%(n=20)$ de los pacientes de la muestra, tanto en la fase oral como en la faríngea presentando un promedio de $0,6 \mathrm{seg}$ para la primera fase y de 0,78 seg para la segunda fase. El $20 \%$ de los casos $(\mathrm{n}=5)$ mostró una prolongación de la duración de la fase oral de $0,74 \mathrm{seg}$ y $1,07 \mathrm{seg}$ para la fase faríngea (Tabla 5). El análisis de los tiempos totales promedios de los 25 pacientes evaluados mostró un promedio de $0,63 \mathrm{seg}$ para la fase oral y $0,84 \mathrm{seg}$ para la fase faríngea (Tabla 6).

Se estableció como normalidad 0,5-0,6 seg para la fase oral y de $0,7-0,8$ seg para la fase faríngea.

Se observó reflujo hacia rinofaringe en un solo caso de 25 , representando un porcentaje de $4 \%$.

Tabla 5. Estudio de la deglución. Resultados para las fases oral y faríngea en números absolutos

\begin{tabular}{lcc}
\hline & Fase oral & Fase faríngea \\
\hline Normal & 20 & 20 \\
Prolongada & 5 & 5 \\
\hline
\end{tabular}

Tabla 6. Fases oral y faríngea de la deglución medidas en segundos

\begin{tabular}{lcccccc}
\hline & Intento 1 & $\begin{array}{c}\text { Fase oral (seg.) } \\
\text { Intento 2 }\end{array}$ & Prom. & Intento $\mathbf{1}$ & $\begin{array}{c}\text { Fase faríngea (seg.) } \\
\text { Intento 2 }\end{array}$ & Prom. \\
\hline Paciente 1 & 0,65 & 0,64 & 0,64 & 0,7 & 0,8 & 0,75 \\
Paciente 2 & 0,62 & 0,63 & 0,62 & 0,8 & 0,8 & 0,8 \\
Paciente 3 & 0,6 & 0,63 & 0,61 & 0,8 & 0,73 & 0,76 \\
Paciente 4 & 0,57 & 0,64 & 0,6 & 0,76 & 0,8 & 0,78 \\
Paciente 5 & 0,74 & 0,69 & 0,82 & 1,2 & 0,9 & 1,05 \\
Paciente 6 & 0,55 & 0,64 & 0,59 & 0,8 & 0,8 & 0,8 \\
Paciente 7 & 0,59 & 0,64 & 0,61 & 0,76 & 0,78 & 0,77 \\
Paciente 8 & 0,6 & 0,61 & 0,6 & 0,77 & 0,82 & 0,8 \\
Paciente 9 & 0,73 & 0,71 & 0,72 & 1,3 & 1 & 1,15 \\
Paciente 10 & 0,59 & 0,64 & 0,61 & 0,81 & 0,82 & 0,81 \\
Paciente 11 & 0,62 & 0,59 & 0,6 & 0,8 & 0,81 & 0,8 \\
Paciente 12 & 0,64 & 0,6 & 0,62 & 0,77 & 0,83 & 0,8 \\
Paciente 13 & 0,6 & 0,6 & 0,6 & 0,79 & 0,83 & 0,81 \\
Paciente 14 & 0,6 & 0,6 & 0,6 & 0,77 & 0,83 & 0,8 \\
Paciente 15 & 0,75 & 0,71 & 0,73 & 1,1 & 0,9 & 1 \\
Paciente 16 & 0,61 & 0,59 & 0,6 & 0,7 & 0,81 & 0,76 \\
Paciente 17 & 0,62 & 0,6 & 0,61 & 0,79 & 0,83 & 0,81 \\
Paciente 18 & 0,63 & 0,6 & 0,61 & 0,81 & 0,8 & 0,8 \\
Paciente 19 & 0,64 & 0,6 & 0,62 & 0,77 & 0,83 & 0,8 \\
Paciente 20 & 0,62 & 0,61 & 0,61 & 0,77 & 0,82 & 0,79 \\
Paciente 21 & 0,57 & 0,65 & 0,61 & 0,8 & 0,77 & 0,78 \\
Paciente 22 & 0,76 & 0,72 & 0,74 & 1,2 & 1,1 & 1,15 \\
Paciente 23 & 0,58 & 0,66 & 0,62 & 0,77 & 0,78 & 0,77 \\
Paciente 24 & 0,75 & 0,71 & 0,73 & 1,1 & 0,9 & 1 \\
Paciente 25 & 0,59 & 0,64 & 0,61 & 0,76 & 0,78 & 0,77 \\
Total & & & 0,63 & & & 0,84 \\
\hline
\end{tabular}


Tabla 7. Relación entre tipo de defecto y número de pacientes con evaluación deglutoria

\begin{tabular}{lcccc} 
& & Fase Oral & Deglución & \multicolumn{2}{c}{ Fase Faríngea } \\
& Normal & Prolongado & Normal & Prolongado \\
\hline Defectos Tipo 2 & 3 & & 3 & \\
Defectos Tipo 3 & 17 & & 17 & 2 \\
Defectos Tipo 4a & & 2 & & 3 \\
Defectos Tipo 4b & & 3 & & \\
\hline
\end{tabular}

Al correlacionar fase de la deglución con tipo de defecto reconstruido según la clasificación propuesta, se observó que aquellos defectos de mayor complejidad (Tipos 4a y 4b) tuvieron alteración de las dos fases de la deglución analizadas, las cua-

Tabla 8. Resultados fonoaudiológicos

\begin{tabular}{lrr} 
& \% & n \\
\hline Excelente & 71 & 18 \\
Bueno & 21 & 5 \\
Aceptable & 8 & 2 \\
Malo & 0 & 0 \\
Total & 100 & 25 \\
\hline
\end{tabular}

les se tradujeron en prolongación de ambas en tiempos de 0,74 seg para la fase oral y 1,07 seg para la fase faríngea (Tabla 7).

\section{c) Evaluación Fonoaudiológica}

Un fonoaudiólogo en Italia y 1 en Chile evaluaron a los 25 pacientes que se les aplicó la encuesta de calidad de vida, utilizando los elementos fonéticos citados en el material y método. El control fonoaudiológico se realizó un año después de la cirugía reconstructiva y se obtuvo que un $71 \%$ de los pacientes, presentó un resultado fonético Excelente, $21 \%$ fue Bueno y un $8 \%$ Aceptable; en opinión del grupo no hubo resultados malos en ninguno de los 25 casos estudiados (Tabla 8).

El análisis fonético reveló que aquellos pacientes de la muestra con defectos tipo 3 y 4 mostraron resultados fonéticos buenos o aceptables (Tabla 9).

Tabla 9. Relación entre tipo de defecto y número de pacientes con evaluación fonética

\begin{tabular}{lccc} 
& Excelente & Resultados Fonéticos \\
& Bueno & Aceptable & Malo \\
\hline Defectos Tipo 2 & 3 & & \\
Defectos Tipo 3 & 14 & 3 & 2 \\
Defectos Tipo 4a & & 1 & 2 \\
Defectos Tipo 4b & & 1 & \\
\hline
\end{tabular}

\section{Discusión}

La reconstrucción Microquirúrgica de grandes defectos producidos por extensas resecciones oncológicas, hoy es posible gracias a que existen los materiales adecuados y los equipos entrenados para hacerlo ${ }^{13}$. El problema radica en el sitio a reconstruir; en este sentido la orofaringe presenta una disposición tridimensional que la hace particularmente compleja, además se agrega el elemento extra que es la selección del colgajo seleccionado y finalmente el tipo de técnica a utilizar.

Varios autores concuerdan en el hecho que, de ser posible una adecuada resección y reconstrucción inmediata, la cirugía debe cumplir un rol principal ${ }^{14}$, sin embargo, existen también detracto- 
res que proponen la radioterapia y/o radioquimioterapia como los tratamientos de elección para esta región ${ }^{15,16}$. No existe consenso al respecto hasta hoy en día como se puede observar al analizar diferentes líneas guías de tratamiento.

De todas las posibles clasificaciones actualmente usadas, la más conocida probablemente sea la de Kimata, quien al dividir en tres los defectos de la unión velo tonsilar sólo plantea el problema desde un punto de vista bidimensional; por el contrario, impresiona que el problema es tridimensional y por esto se requiere de un enfoque espacial para interpretar adecuadamente el defecto a reconstruir y el tipo de colgajo a utilizar. En este sentido el colgajo libre radial de antebrazo posee características especiales de plegabilidad, grosor y extensión que permiten una adecuada reconstrucción; sin embargo, es además necesario diseñar este colgajo en función al defecto tridimensional creado, ya que no todos los defectos espacialmente son iguales.

Especial interés reviste el diseño del colgajo radial de antebrazo que tradicionalmente ha sido rectangular, de hecho en los defectos tipo 2 y 3 de la clasificación propuesta, este fue el que se utilizó con variaciones en cuanto a su plegabilidad espacial, sin embargo, en los defectos tipo 4 es donde se refleja mejor el enfoque tridimensional del problema reconstructivo, por esta razón se realizó una variación en el diseño que consistió en un colgajo tipo "P" de manera tal que el pie de ésta se posicionara sobre la pared lateral de la orofaringe y la cabeza permitiera reconstruir el defecto del velo del paladar, esta variación permitió en definitiva poder jugar con los ejes espaciales y en particular con las líneas de tensión disminuyendo de esta forma el número de dehiscencias que se observan en otras series 5 .

Es necesario además pensar que el esfuerzo reconstructivo debe necesariamente tener una traducción que permita una conservación de las diferentes funciones en las cuales participa esta región, es decir, en la fonación y en la deglución y por ende en la calidad de vida del paciente.

La elección de una serie quirúrgica prospectiva y longitudinal permitió evaluar a partir de un punto inicial una clasificación quirúrgica y asociarla con un procedimiento reconstructivo midiendo sus resultados en el tiempo. De la misma manera resolvió algunos problemas temporales, geográficos y de análisis de datos, en contraposición con un estudio de casos y controles que probablemente habría planteado además un problema de tipo ético.

Tal vez un estudio que comparara dos ramas (cirugía con radioterapia o radioquimioterapia) aportaría más información en cuanto a resultados de un tipo particular de terapia, sin embargo, el objetivo de esta experiencia era uno distinto.

En esta serie se observó que en un reducido porcentaje de los pacientes existió alteración de la fonación o de la deglución en forma importante, en tanto que la calidad de vida obtuvo en general buenos o excelentes resultados.

En relación a la evaluación de la calidad de vida, se aplicaron los cuatro criterios básicos determinados por el Dr. Gliklich $\mathrm{RE}^{8}$ y que comprenden dolor, alimentación, lenguaje y aspecto; es interesante este punto ya que el esfuerzo por determinar una escala que sea representativa sólo de la patología de cabeza y cuello, resulta del entrecruzamiento de la información que aportan otros cuatro sistemas de evaluación (UW-QOL v 4.0, SF-36 Health Survey, the H\&NS y el PSS-HN), sin embargo, es necesario señalar que para la aplicación estándar de esta escala se debe realizar un estudio dirigido para su validación idiomática. Es importante mencionar además que la aplicación por parte de un solo encuestador para la aplicación de los parámetros tiene un posible sesgo derivado del hecho que es el mismo autor quien hizo la medición, probablemente esto debiera ser corregido en estudios posteriores.

Especial interés merece el estudio de la deglución que se realizó por cinefluoroscopía dado que es la técnica más accesible tanto en el sistema público italiano como el chileno y las dosis de radiación, a pesar de tratarse de dos tomas consecutivas son de tan baja intensidad que permiten realizar esta técnica exponiendo al paciente a un riesgo mínimo; si bien existen otros estudios diagnósticos se consensuó en ésta por la fácil reproducibilidad.

$\mathrm{Al}$ analizar por tipo de defecto, fue en los de tipo 3 y 4 donde mayoritariamente se vieron más afectadas estas funciones; al realizar una comparación con otras publicaciones se observa que los porcentajes de complicaciones y resultados funcionales son inferiores a nuestra serie si consideramos los mismos subsitios reconstruidos, como por ejemplo en las series en donde se analizan los métodos de reconstrucción tipo Jump y Denude ${ }^{5,17}$.

Del análisis de los casos de la serie, se desprende que tanto la viabilidad del colgajo como los resultados funcionales hacen de esta clasificación un instrumento que permite una adecuada reconstrucción de esta área anatómica, sin embargo, es necesario hacer el alcance que, dado que el seguimiento y evaluación de la muestra fue sólo de un $67,56 \%$, tal vez lo más correcto sería plantear esta clasificación reconstructiva como una propuesta innovadora que muestra las observaciones preliminares de una técnica, pero que crea la necesidad de una rigurosidad más acuciosa en la recolección de los datos que permitan una evaluación en el tiempo de los pacientes. 


\section{Referencias}

1. Podrecca S. Review of 346 patients with free-flap reconstruction following head and neck surgery for neoplasm. J Plast Reconstr Aesthet Surg 2006; 59: 122-129.

2. Riggio E, Parafioriti A, Tomic O, Podrecca S, Nava M, Colombetti A. Experimental study of a sleeve microanastomotic technique. An Plast Surg 1999; 43: 625-631.

3. Song R. The forearm flap. Clin Plast Surg 1982; 9: 21-26.

4. Soutar D. The radial forearm flap: a versatile method for intraoral reconstruction. Br J Plast Surg 1983; 36: 1.

5. Kimata Y. Velopharyngeal function after microsurgical reconstruction of lateral and superior oropharyngeal defects. Laryngoscope 2002; 112: 1037-1042.

6. Recamán M. Reconstrucción del esfínter velo faríngeo en la fisura del paladar secundario: alternativa quirúrgica. Cir Pediátrica 2006; 19: 106-110.

7. Dodds WJ. Physiology and radiology of the normal oral and pharyngeal phases of swallowing. AJR 1990; 154: 953-963.

8. Gkliklich R. Are Head and neck specific quality of life measures necessary? Head and Neck 1997; 19: 474480.

9. Logemann J. Swallowing physiology and pathophysiology. Otolaryngol Clin North Am 1988; 21: 613-623.

10. Logemann JA Swallowing disorders in three types of head and neck surgical patients. Cancer 1979; 44: 1075-1082.

11. Pauloski BR. Surgical measures affecting swallowing in patients treated for oral/oropharyngeal cancer. Head and Neck 2004; 26: 625-636.

12. Skelly M. Changes in phonatory aspects of glossectomy intelligibility through vocal parameter manipulation. J Speech Hear Disord 1972; 37: 379-389.

13. Seikaly H. Functional outcomes after primary oropharyngeal cancer resection and reconstruction with the radial forearm free flap. Laryngoscope 2003; 113: 897-904.

14. McMillan A. Preservation of quality of life after intensity-modulated radiotherapy for early-stage nasopharyngeal carcinoma: Results of a prospective longitudinal study. Head and Neck 2006; 28: 712 722.

15. Pérez CA, Carmichael T, Devineni VR. Carcinoma of the tonsillar fossa: a non randomized comparison of irradiation alone or combined with surgery-long-term results. Head and Neck 1991; 13: 282-290.

16. Lee WR, Mendehall WM, Parsons JT, Million RR, Cassisi NJ, Stringer SP. Carcinoma of the tonsillar region: a multivariate analysis of 243 patients treated with radical radiotherapy. Head and Neck 1993; 15: 283-288.

17. Gehanno P, Guedon C, Veber P, Perreau P, Alalout P, Moisy N. Rehabilitation velo-Pharygee apress bucco pharyngectomie-transmaxillaire elargie a la region velo-palatine. Ann Otolaryngol Chir Cervicofac 1985; 102: $135-137$. 


\section{INFORME TRABAJO DE INGRESO}

Quiero agradecer al directorio de la Sociedad de Cirujanos de Chile por la distinción que se me ha otorgado, de informar el trabajo de ingreso "Reconstrucción Microquirúrgica del Velo del Paladar y Pared lateral de Orofaringe. Una Nueva Propuesta de Clasificación y Resultados Funcionales".

En el año 1906 el Dr. Crile presentó su trabajo referente a los vaciamientos cervicales radicales en tumores de cabeza y cuello, técnica que, en esa época, tuvo una alta morbimortalidad, y por esta razón quedó prácticamente abandonada hasta que en 1933 Fishel demostró científicamente que esta técnica permitía tener mejores tasas de sobrevida en pacientes portadores de cáncer de cabeza y cue1lo. Posteriormente en la década del 40 Hayes Martin definió las bases de la cirugía oncológica de cabeza y cuello que conocemos hasta hoy en día. Desde la década del 40 hasta hoy las tasas de sobrevida han mejorado discretamente, sin embargo, ha habido un enorme crecimiento en lo que se refiere a técnicas reconstructivas, rehabilitación, técnicas de conservación de órgano; en el último tiempo se han realizado una serie de estudios para determinar el impacto en la calidad de vida de los pacientes sometidos a diversas terapias, que a fin de cuenta es lo que importa después de salvar la vida del paciente con cáncer.

El trabajo del Dr. Compan aborda un tema relacionado con el área reconstructiva y la calidad de vida de los pacientes.

Se presenta una serie de pacientes portadores de cáncer de orofaringe, en los cuales se realizó una resección oncológica y posteriormente una reconstrucción con un ingenioso diseño de colgajo libre microvascularizado.

$\mathrm{La}$ orofaringe es un territorio extremadamente complejo desde el punto de vista anatómico y funcional, y la historia ha demostrado que intentos resectivos con criterio oncológico sin una reconstrucción adecuada, dejan graves secuelas funcionales en la deglución y el habla. En la actualidad sabemos que la enfermedad en estadios tempranos, tiene tasas de sobrevida similares si el tratamiento es quirúrgico o con radioterapia, sin embargo, en estadios avanzados III y IV entran en juego terapias combinadas como cirugías más radioterapia, o radioquimioterapia adyuvante, o protocolos de conservación de órgano en esquemas de radioquimioterapia. En la planificación de la estrategia terapéutica también influye el subsitio oncológico que se esté tratando, por ejemplo en base de lengua y pared posterior de faringe hay una tendencia a optar por tratamiento quirúrgico como primera línea, en cambio en paladar blando, amígdala y pared lateral la mayoría de los centros prefieren tratamientos radiantes o protocolos de conservación de órgano.

La forma de medir los resultados es a través de curvas de sobrevida, y cuando las curvas de sobrevida se mantienen estables se debe medir la satisfacción de los pacientes y calidad de vida. Es por este motivo que en el último tiempo han aparecido una serie de publicaciones en las cuales los pacientes se ven sometidos a test de calidad de vida, lo cual se ha transformado en un estudio estándar de vital importancia para poder evaluar y comparar nuestros resultados.

La serie presentada por el Dr. Compan se refiere a pacientes con cáncer de orofaringe, los cuales fueron seleccionados según estrictos criterios para ingresar a un protocolo de estudio en el cual se aplicaría una resección oncológica más una reconstrucción con un colgajo microvascularizado antebraquial diseñado según la necesidad anatómica tridimensional del defecto. Este punto es probablemente el aporte más interesante de este estudio, ya que plantea una hipótesis señalando que las actuales reconstrucciones y clasificaciones propuestas no satisfacen las necesidades de los defectos creados por la acción quirúrgica, y propone un modelo reconstructivo nuevo e ingenioso, según el tipo de defecto tridimensional, para lo cual también propone una clasificación ad-hoc. Posteriormente, hace una evaluación funcional de la reconstrucción con un instrumento fonético para evaluar el habla y un método radiológico para evaluar la deglución. Y finalmente, realiza una encuesta de calidad de vida a parte del grupo.

Sus resultados demuestran que el diseño del colgajo cumplió las expectativas en lo que se refería a las necesidades de reconstrucción según la clasificación propuesta, validándola. El colgajo diseñado resultó satisfactorio para lograr la reconstrucción tridimensional planificada con una tasa baja de complicaciones. En el análisis de los resultados, de la aplicación de test funcionales y de calidad de vida, estos parecen a simple vista promisorios, sin embargo, la pérdida de una parte importante de la muestra $(32 \%)$, hace que estos resultados sean imposibles de validar, lo que es honestamente admitido por el autor en los comentarios finales, y que, lamentablemente, quedará pendiente para poder consolidar definitivamente la técnica. Como cirujano de 
cabeza y cuello creo que sería interesante saber algunas otras cosas de este trabajo como: vías de abordajes, estadios en los cuales se puede aplicar la técnica, sobrevidas, comparación con otros esquemas terapéuticos, calidad de vida en grupos comparados en diferentes modalidades de tratamiento, etc, todo con el fin de poder incorporar la técnica a nuestro armamentario terapéutico. Quiero nuevamente rescatar que este trabajo nos muestra una alternativa terapéutica reconstructiva ingeniosa y novedosa, y una clasificación que puede ser útil al momento de decidir la mejor técnica reconstructiva, en un área altamente conflictiva y de difícil manejo.

Debemos acostumbrarnos a validar científicamente nuestro trabajo, ya que estamos en la mira permanente de nuestros pares, residentes y alumnos, debido a la incorporación de técnicas de análisis científico cada vez más exigentes, encuestas e ins- trumentos de medición y estrictos marcos legales. Quiero aprovechar esta tribuna para agradecer las enseñanzas de mis residentes en lo que respecta al permanente análisis crítico de la literatura, el cual me permite mejorar notablemente el trabajo científico y refrescar continuamente mi espíritu.

Estoy seguro que con estas observaciones el Dr. Compan podrá completar, a corto plazo, su estudio y responder las dudas que aun nos quedan.

Señores del directorio de la Sociedad de Cirujanos, en base al mérito de este trabajo, me permito sugerir el ingreso del Dr. Compan a la Sociedad de Cirujanos de Chile.

Dr. Felipe Capdeville F. Instituto Nacional del Cáncer, Clínica Alemana Santiago, Chile 